\title{
IV. Wissenschaftstypen als Tiefenstruktur interdisziplinärer Zusammenarbeit
}

\section{Typologie der Wissenschaften: Neun Wissenschaftstypen als Typen mit Familienähnlichkeiten}

Die Gegenstände, die unter einen Begriff fallen, sind nicht durch Eigenschaften verbunden, die allen gemeinsam wären, sondern durch ein Netz von >Familienähnlichkeiten<. Das war die Einsicht, mit der Ludwig Wittgenstein das Begriffsdenken im 20. Jahrhundert revolutionierte. Damit etwas z.B. Spiel heißen kann, muss es nicht bestimmte allgemeine Eigenschaften aufweisen (z.B., dass man um den Sieg spielt oder mit anderen), sondern nur hinreichend viele Gemeinsamkeiten mit einem Fall, der als Spiel gilt. So können wie bei einem Tau, durch das sich kein einziger durchgängiger Faden durchzieht, aber jeder jeweils andere streckenweise übergreift, Phänomene unter einen Begriff fallen, die nur über Zwischenglieder mit anderen verbunden sind. ${ }^{1}$

Mit dieser Auffassung vom Begriff lässt sich auch das Problem der Einheit und Vielheit in den Wissenschaften auflösen. Wissenschaft lässt sich so verstehen als eine Familie von Fällen mit unterschiedlichen, einander teils mehr, teils weniger überlappenden Ähnlichkeiten. Die Wissenschaftstypen zeigen sich durch unterschiedliche Verwandtschaften miteinander verknüpft. Unter dem einen oder anderen Gesichtspunkt kann der eine oder andere Typus als prototypisch erscheinen - jedoch: diese Gesichtspunkte lassen sich wechseln, sodass das Gesamtbild sich jeweils umstellt. Im Wech-

1 Ludwig Wittgenstein: Das Blaue Buch. In: Werkausgabe Bd. 5, hrsg. v. Gertrude Anscombe/E. M. Gertrude/Rush Rhees/Georg H. Wright, Frankfurt/M 1984, S. 37; Ludwig Wittgenstein: Philosophische Untersuchungen, In: Werkausgabe Bd. 1, Frankfurt a.M. 1982, §66. 
sel der Perspektiven zeigen sich unterschiedliche Wissenschaftstypen als gleichwertige Motivations-, Praxis- und Zugangsformen.

Die historische Darstellung offenbarte, dass jeder Forschungstyp in seiner Geschichte motiviert ist und eigene Validierungsmethoden besitzt. Ob z.B. eine Rekonstruktion als wissenschaftliches Ergebnis zählen kann, hängt davon ab, ob die verfügbaren Relikte, Fragmente oder Daten sich >stimmig< in ein Ganzes fügen lassen, das die Form und Beschaffenheit der zusammengesetzten Elemente miterklärt; ob eine Beobachtung oder Erhebung wissenschaftlich valide ist, hängt davon ab, ob die Zählung, Messung, Datierung oder Beschreibung Gegebenheiten von überlagernden Erscheinungen abzulösen, zu sidentifizieren zutragen vermag; ob experimentelle Forschung wissenschaftliche Ergebnisse zeitigt, ist soweit der Fall, als es gelingt, ein Experimentalsystem zu stabilisieren, dass in der kontrollierten Variation ssignifikante Unterschiede erzeugt in Bezug auf theoretisch beschreibbare Strukturen (seien diese Beschreibungen sprachlich oder in einem formalen Symbolismus verfasst, z.B. in der chemischen Formelschreibweise).

Jeder Typus von Forschung besitzt im Frage-Antwort-Zusammenhang von Wissenschaft eine eigene Struktur und einen eigenen Wert. Dabei fügen sich die Wissenschaftstypen nicht etablierten Disziplingrenzen. Nehmen wir die Wissenschaftsform der Kritik: die Reflexion auf Erkenntnis und ihre Bedingungen ist längst nicht mehr nur ein philosophisches Unternehmen, das eigene Validierungskriterien enthält (z.B. ob sich die Darstellung schlüssig auf sich selbst anwenden lässt), sondern auch ein wissens- und wissenschaftssoziologisches, ein historiographisches, ein anthropologisches und ethnographisches; es findet sich überall da, wo kritische Diskurse verwurzelt sind, die subjekt-, ideologie-, historisch-, regional-, gender-, disziplin- oder klassenbedingte Wirkungen in der Erkenntnis reflektieren. Ebenso ist z.B. die Wissenschaftspraxis Rekonstruktion konstitutiver Bedingungen eines Phänomens ebenso forschungsleitend in der Prähistorischen Archäologie (wenn etwa das Phänomen eines um 400000 v. Chr. (sic!) auf exzellente Flugfähigkeit hin designten Speeres die Frage aufwirft, welche sozialhistorischen und anthropologischen Bedingungen dafür vorausgesetzt werden müssen) ${ }^{2}$

2 Vgl. H. Thieme (Hrsg.): Die Schöninger Speere-Mensch und Jagd vor 400.000 Jahren, Stuttgart 2007. 
wie in der Vulkanologie (wenn danach gefragt wird, welches Zusammenspiel geologischer Faktoren einen ungewöhnlichen Ausbruch hervorbrachten).

Wir finden also eine Reihe von Forschungstypen vor, die jeweils einen Eigenwert besitzen und - wie die historische Rekonstruktion zeigte - tief verankerte und historisch gewachsene Ideale von Wissenschaftlichkeit verkörpern. Konflikte um Wissenschaft treten immer da auf, wo dieser jeweilige Eigenwert nicht geachtet wird und wo ein Forschungstypus zur eigentlichen, wahren Form von Wissenschaft erklärt wird. Ob es die logische Beweisform, der technische Erfolg, die Objektivität der brute facts, die Überschreitung des Alltagsverständnisses, die Reflexionstiefe der Argumentation oder die Bewältigung von Datenmengen ist: Jede wissenschaftliche Leistung kann auf den Thron der Wissenschaftlichkeit gehoben und ihr gegenüber alle anderen entwertet werden. Eine solche Vorrangstelle ist aber nicht nur unbegründet, sie verhindert auch interdisziplinäre, genauer: interwissenschaftstypische Kooperation auf Augenhöhe. Das heißt: Sie verhindert, dass jeder Forschungsansatz in einer Kooperation seine eigenen Stärken vollständig einbringen kann.

Wissenschaft als eine Familie von Typen zu begreifen, bedeutet nicht, dass sich diese Typen einem Vergleich untereinander entziehen. Wie auch für Familienmitglieder Vergleichsgrößen bestehen (Haarfarbe, Nasenform, Körperbau, Augenfarbe etc.), so gibt es auch für die Wissenschaftstypen Größen, in Bezug auf die sie sich vergleichen lassen - wodurch sich die Familienähnlichkeiten allererst ja zeigen. Mithilfe solcher Vergleichsgrößen lassen sich Gemeinsamkeiten und Unterschiede aufzeigen, ohne diese Unterschiede als Unterschiede des Wertes oder der Entwicklung zur Wissenschaftlichkeit aufzufassen. Sie sind als rein typologische Unterschiede zu begreifen.

Das bedeutet methodologisch, dass nur neutrale und wertfreie Vergleichsinstanzen zum Einsatz kommen können. Ein solches Tertium Comparationis muss ausreichend struktural und praxisbezogen angelegt sein, es darf nicht ein spezifisches Wissenschaftsideal abbilden.

Als Vergleichsinstanzen in diesem Sinne bieten sich an:

1. die Art der Fragen, die einen Wissenschaftstypus kennzeichnen

2. die Praktiken, die ihn charakterisieren

3. die Form dessen, was in einem Typus als wissenschaftliches Ergebnis gilt 
In diesen drei Hinsichten lassen sich neun Grundkonzeptionen von Wissenschaft voneinander unterscheiden:

1. Gegenstandserfassender Forschungsansatz: Daten zu erheben, ist eine Grundform von Wissenschaft. Wie werden Daten erhoben? Indem fragliche Elemente beobachtet, gezählt, gemessen, datiert, verortet oder beschrieben werden. Die einfachste Form besteht in Fragen der Art: Wie häufig tritt $\mathrm{x}$ auf? Wo tritt $\mathrm{x}$ auf? Wann tritt $\mathrm{x}$ auf? Mit Instrumenten lassen sich Fragen nach Temperatur, Masse, elektrische Leitfähigkeit, Alter eines Elementes verfolgen. Aufzeichnungssysteme wie Zeitstrahlen, Karten, Tabellen und Diagramme dienen der Erfassung der Daten.

2. Typisierend-ordnungsgenerierender Forschungsansatz: Während in der gegenstandserfassenden Variante das Element $\mathrm{x}$ selbst nicht infrage steht, wenn z.B. ein Planet verortet oder eine Chronologie von Herrschern erstellt wird, stellt sich im typisierend-ordnungsgenerierenden Ansatz die Frage nach der Bestimmung von x: Welchem Typ, welcher Art, welcher Gattung, welcher Epoche, welchem Genre ist x zuzuordnen? Die Basis-Praxis dieses Forschungsansatzes ist das Vergleichen: Welche Merkmale finden sich im einen Exemplar, welche im anderen? Welche Typen sind $\mathrm{zu}$ unterscheiden? Welche Typen sind allgemeiner, welche spezieller? Wie ist das Ordnungssystem als Ganzes aufgebaut? Ergebnisse finden hier üblicherweise ihre Darstellungen in Klassifikationsschemata, komparatistischen Erörterungen und synoptischen Taxonomien.

3. Auch die klassische Statistik bewegt sich in ihrer Grundform im Rahmen dieses Forschungsparadigmas. In der statistischen Variante werden Vorkommnisse nicht nur typisiert, sondern zugleich in ihrer Häufigkeit erfasst. Damit eröffnet sie ein ganz eigenes Forschungsfeld: das der Häufigkeitsverteilung. Es lassen sich nun Häufigkeiten von Ereignissen in Bezug auf andere Ordnungsfragen vergleichen: Wie sieht es in welcher Zeit aus? Wie in welcher Gegend? Wie bezogen auf welche Bevölkerungsanteile etc.? Statistik produziert damit ein Wissen zweiter Ordnung: ein $\mathrm{x}$ wird als Gegenstand zuordnend erfasst (erste Ordnung) und sodann in seiner Häufigkeit in Hinsicht auf ein zweites Einteilungssystem in vergleichende Darstellung gebracht (zweite Ordnung). Statistisches Wissen ist zum allergrößten Teil tabellarisches und diagrammatisches Wissen.

4. Rekonstruierender Forschungsansatz: Die Grundfrage hier ist: Wie sah ein Ganzes aus, wenn nur Relikte, Indizien, Spuren, Fragmente vorhanden 
sind? Die Forschungsform ähnelt hier der Untersuchung eines Kriminalfalls: ein Ablauf, ein Geschehen, ein Zusammenhang soll als eine schlüssige Ganzheit rekonstruiert werden. Das kann dann in der Form eines Rekonstruktionsmodells wie etwa in der Archäologie (materiell oder digital), in Form einer Narration wie in manchen geschichtswissenschaftlichen Studien oder in Form einer Strukturbeschreibung geschehen. Die bestimmende Praxis ist hier die versuchsweise Kombination im Sinne von: Wie passt $\mathrm{x}$ mit y zusammen? Was ergäbe sich für das Ganze, wenn $\mathrm{x}$ als a und $y$ als $b$ betrachtet würde? Und wenn das Ganze so oder so aussähe, dann wäre wiederum das Element $\mathrm{z}$ als $\mathrm{c}$ zu betrachten; kann das sein? Diese Frageform des Rätsellösens in einem Teil-Ganzes-Setting verbindet - in ihrer Form, nicht im Gegenstand - historische Rekonstruktion mit Textauslegung und manchen Formen der Experimentalforschung: In jedem Fall versucht man, am Einzelnen eine sTheorie « über einen Gesamtzusammenhang zu entwickeln und an einem (vorausgesetzten oder hypothetisch entworfenen) Gesamtzusammenhang Verhalten und Beschaffenheit von Elementen als integrale Bestandteile zu deuten.

5. Rekonstruktiv-erklärender Forschungsansatz: Sind nicht Indizien, Spuren und Relikte gegeben, die auf ihren vormaligen Zusammenhang hin gedeutet werden müssen, sondern ein als solches gegebenes oder vorausgesetztes Phänomen, z.B. die Entstehung einer Revolution, das überraschende Verhalten eines chemischen Stoffes, der Klimawandel, eine Krankheit o.ä., dann richtet sich die Forschung auf das Bedingungsgefüge, unter dem das Zustandekommen von x zu erklären ist. Was muss als gegeben angenommen werden, dass das (erstaunliche, fragwürdige) Phänomen x zustande kommt? Die Forschung richtet sich hier auf die in einem Bedingungsgefüge zusammenwirkenden Faktoren, die am Entstehen einer Ganzheit, eines Phänomens in je zu bestimmender Weise beteiligt sind. Weil ein solches Bedingungsgefüge, z.B. für den Klimawandel, für ein historisches Ereignis oder für das Entstehen von menschlicher Sprache extrem komplex sein kann, hat auch hier die Forschung einen stark heuristischen und experimentellen Charakter. Zum Einsatz kommen unterschiedliche Mittel der Modellierung, die das Bedingungsgefüge probeweise zusammenstellen und Abläufe, die infrage stehen, probeweise simulieren. Solche Modellierungen werden typischerweise auf komplexe Naturvorgänge angewandt, aber auch immer häufiger in Sozialwissenschaften eingesetzt. 
6. Der Forschungsansatz der interpretativen Adäquation: Während im Labor ausdrücklich mit künstlichen Bedingungen gearbeitet wird, gewinnt die Forschung im Paradigma der Adäquatheit ihre Validität dadurch, dass sie den Phänomenen in ihrem Aspektreichtum durch Anpassung der Darstellungsmittel möglichst umfassend gerecht $\mathrm{zu}$ werden sucht. In einer Linie mit dem Gedanken der adaequatio intellectu et rei kondensiert sich die Bemühung um die Anpassung der seigenen vorausgesetzten Konzepte und Darstellungsmittel an den Gegenstand in einem eigenen Forschungsansatz. Die leitende Frage lautet: Wie müssen Begriffe, Konzepte, Regelformulierungen verändert werden, damit sie einen Gegenstand in seiner Phänomenalität zum Vorschein bringt, ohne konstitutive Elemente und Aspekte zu vernachlässigen? Wenn z.B. in der Ethnologie nach der Bedeutung einer sozialen Praxis gefragt wird oder in der Philologie nach der angemessenen Übersetzung eines altsprachlichen Wortes, dessen Bedeutungsaspekte aus einer Fülle von Kontexten gewonnen worden sind, besteht die Aufgabe darin, eine diesen Aspekten angemessene Interpretation zu geben. Eine angemessene Interpretation wiederum muss nicht in einer passenden Übersetzung münden, tatsächlich ist ein Aufdecken der Übersetzungsproblematik und die Notwendigkeit einer beschreibenden Auslegung das typische Resultat einer auf Adäquation gerichteten Forschung.

7. Der Forschungsansatz der Kritik. Dass jedes Wissen oder Gedachte reflexiv $\mathrm{zu}$ erfassen und zu durchdringen ist und dass es unter je spezifischen Bedingungen zustande kommt, dass zudem die Aufklärung und Reflexion der Bedingungen zumindest ein zentrales Moment von gewissenhafter Forschung darstellt, dies ist das Grundmotiv der kritischen Forschungspraxis. Ihre Praxisform lässt sich als die des Herausschälens, Aufspürens, Entlarvens und Aufdeckens beschreiben. Verborgene, unbemerkte, vernachlässigte oder verdrängte Einflussfaktoren werden detektiert und nach vorn gestellt. Erkenntniskritik, Ideologiekritik und Dekonstruktion sind Namen für Ansätze in diesem Paradigma Kritischer Forschung. Wenn die jeweils aufgedeckten Zusammenhänge als `wahre Motive ‘ hinter dem Schein der Oberfläche aufgefasst werden, hat dieser Ansatz Ähnlichkeiten mit dem Reduktionismus vom analytisch-apodiktischen Typus: In beiden Fällen wird dann der Phänomenbestand als Schein auf das Sein einer wahren Struktur zurückgeführt. Richtet er sich aber auf das Bedingungsgefüge von Erkenntnis, ohne darin universale Gesetzmäßigkeiten 
anzunehmen, dann zeigt er sich als eine genuine Form phänomenbezogener Forschung - eine solche nämlich, die die Erscheinungsweise des Phänomens in seinen Erscheinungsbedingungen offenlegt.

8. Reduktiv-apodiktischer Forschungsansatz: Geht man davon aus, dass sich das Bedingungsgefüge, aus dem sich ein Gesamtzusammenhang erklärt, restlos von jeweiligen Erscheinungen lösen und als universales und gesetzmäßiges Regelwerk in einer formalen Sprache darstellen lässt, dann ergibt sich eine andere Form der Grundfrage: Wie lässt sich ein phänomenaler Zusammenhang auf einen universalen Formalismus zurückführen? Die wissenschaftliche Praxis besteht hier in erster Linie darin, eine formale, logische oder mathematische Sprache so aufzubauen, dass sich in ihr die infrage stehenden Eigenschaften abbilden lassen; man spricht heute gerne von mapping. Eine solche Praxis ist zurzeit am häufigsten im Bereich der Linguistik, der Neurowissenschaften und der Genetik anzutreffen. Gemeinhin ist sie in erster Linie Programm, wie im Bereich der Kognitionswissenschaften. Da diese Wissenschaftsform im Einklang mit den metaphysischen Voraussetzungen der atomistisch-mechanistischen Deutung der Beweislehren steht, die die Wissenschaftsphilosophie lange Zeit geprägt und noch immer starken Einfluss auf Hintergrundüberzeugungen hat, bestimmt sie stärker ein bestimmtes Bild von Wissenschaften als tatsächliche Forschungspraxis.

9. Der Ansatz der experimentellen Laborwissenschaften: In diesem Forschungsansatz werden symbolsystemische Formalismen nicht als Zielpunkt der Forschung eingesetzt, auf den phänomenale Zusammenhänge zurückgeführt werden (Reduktion), sondern als Forschungsinstrumente. Der Formalismus wird im Labor nicht schlicht bestätigt, sondern eingesetzt, um Experimentalsysteme zu strukturieren, die sich darauf richten, noch unbekannte Eigenschaften, Wirkfaktoren und Interdependenzen im Gegenstandsbereich zu entdecken und zu beschreiben. Die zentrale Praxis ist die der Einrichtung von möglichst stabilen Experimentalsystemen, sodass einzelne Faktoren kontrolliert und variiert werden können. Zur Ergebnissicherung gehört hier niemals nur die Angabe eines RegelFormalismus (’Erklärung), sondern stets die Beschreibung des Experimentalsystems, des technischen Versuchsaufbaus und der idealisierten Bedingungen, unter denen sich die Zusammenhänge beobachten ließen. Es ist dieser Forschungstyp, der die Frage der Reproduzierbarkeit aufwirft. 


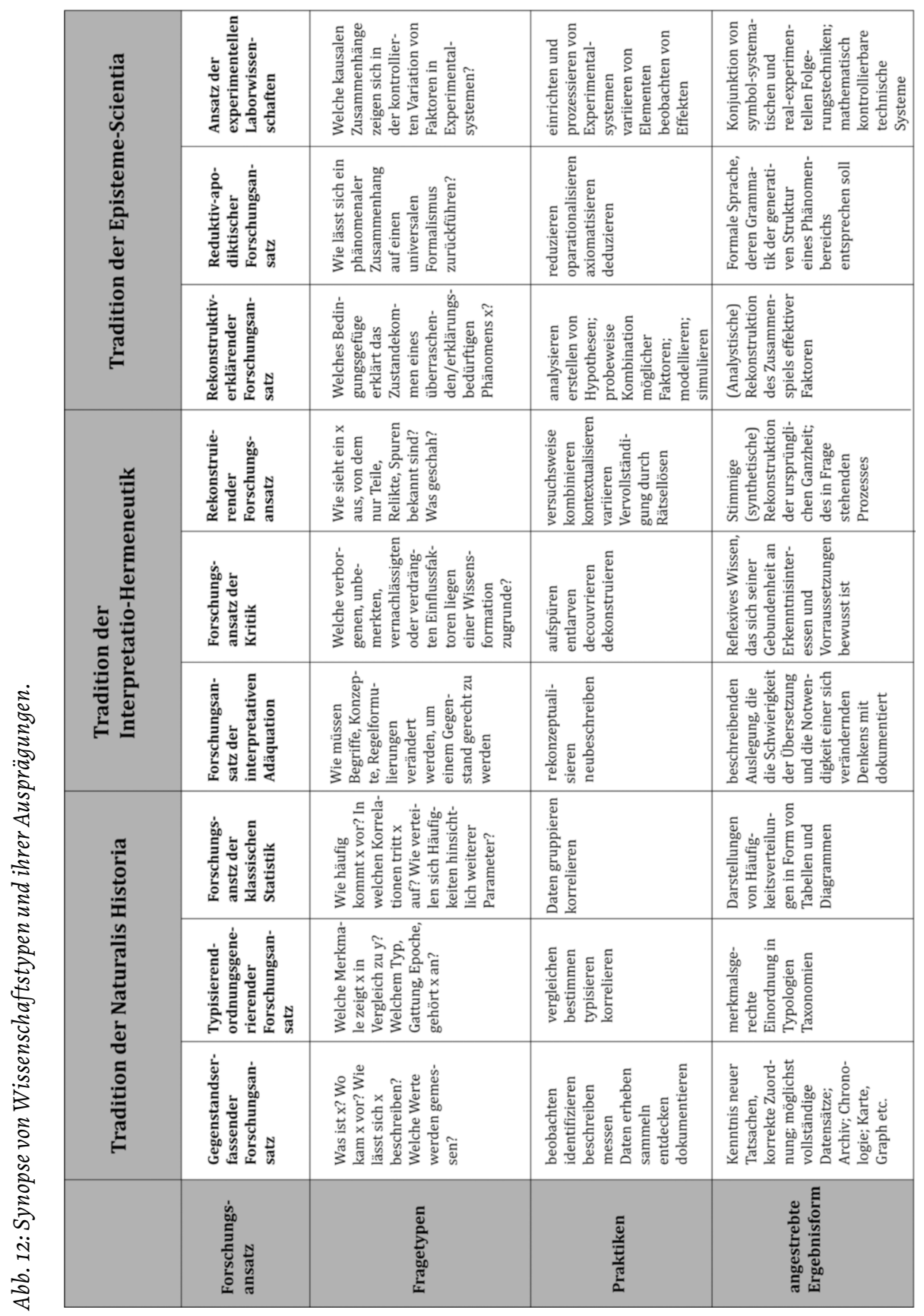


Synoptisch lassen sich somit neun Typen von Wissenschaft in einem Schema nebeneinanderstellen (Abb. 12).

Diese Typologie ist aus historischen Rekonstruktionen gewonnen und stellt eine Möglichkeit dar, Wissenschaftstypen als jeweils eigenständige Praxis- und Motivationskomplexe zu beschreiben. Die Darstellungsform hat den Vorteil, auf einen Blick das Nebeneinander von Typen sichtbar zu machen und sie regt zu Vergleichen an. Sie hat aber auch Nachteile, die in der Bildlogik liegen: Zum einen mag es erscheinen, dass, bedingt durch unsere Leserichtung von rechts nach links, wir es mit einer Folgeordnung zu tun haben, die doch eine Richtung zu höherer Integration und Komplexität und damit eine implizite Hierarchie suggeriert. Zum anderen erschwert die serielle Anordnung Vergleiche von entfernt voneinander angeordneten Wissenschaftstypen. Um diese Nachteile zu umgehen, wäre eine dreidimensionale Darstellung vorzuziehen - etwa in der Art von Abb. 13.

Eine solche freiere Darstellung stellt uns nun vor die Frage nach den möglichen Verhältnissen der Wissenschaftstypen zueinander. Wenn diese Darstellung geeignet ist, ein hierarchisches Bild der Verhältnisse zwischen wissenschaftlichen Motivations-Praxis-Typen abzuwenden und damit die notorischen Kämpfe um Deutungshoheit zu überwinden, dann eröffnet sie die Möglichkeit von Kooperationen auf Augenhöhe: sie verbindet sich nun mit dem Themen- und Aufgabenkomplex der Interdisziplinarität.

Abb. 13: Wissenschaftstypen in nicht-serieller Darstellung.

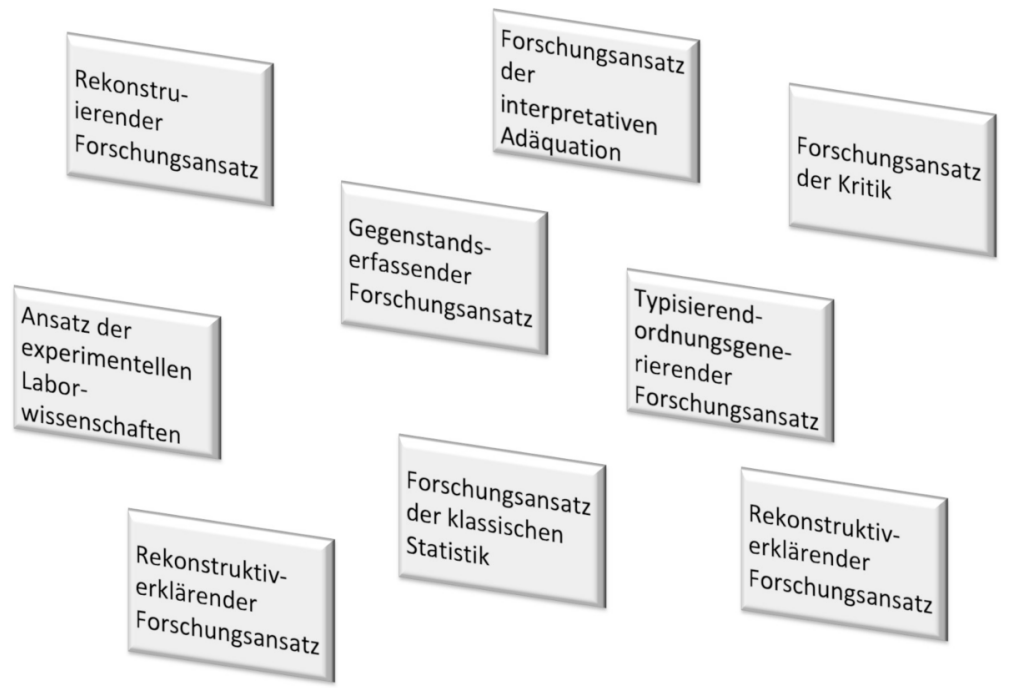




\section{Die Pluralität der Wissenschaften und das Problem der Interdisziplinarität}

Begonnen haben wir mit der Frage, wie es um Einheit und Vielfalt der Wissenschaften steht und angeschlossen haben wir die Frage, wie unter der Bedingung einer Pluralität von Wissenschaftstypen Zusammenwirken gedacht und ermöglicht werden kann. Der klassische Leitbegriff, unter dem dieses Thema verhandelt wird, ist Interdisziplinarität. Die Forderung nach interdisziplinärem Arbeiten durchzieht die gesamte Literatur der Forschungsanträge und wissenschaftsplanerischen Projekt- und Strategiepapiere. Doch in der Praxis erweist sich Interdisziplinarität als Herausforderung. Die damit verbundenen Schwierigkeiten und Konflikte sind seit längerem benannt und aufgelistet. So spricht etwa eine Studie zum Management von Forschungsverbünden ${ }^{3}$ von den Schwierigkeiten interdisziplinärer Zusammenarbeit in deutlichen Worten:

»Einige der Schwierigkeiten erwachsen aus der Tatsache, dass Inter- und Transdisziplinarität eine Kooperation zwischen disziplinären `Kulturen ‘ darstellt. Die Angehörigen dieser disziplinären `Kulturen verfügen über eine je eigene Sprache, die sich von derjenigen anderer Disziplinen und von der Alltagssprache unterscheidet, und sie bedienen sich disziplinenspezifischer Theorien und Methoden. Ebenso haben sie je eigene Vorstellungen über das rrichtige< Vorgehen. Fachsprache, Theorien und Methoden sind Ausdruck einer disziplinenspezifischen Weltsicht und Handlungsweise. ${ }^{4}$

Daraus entstehen in der Darstellung dieser Studie eine Reihe von Konflikten:

»Der Wissenskorpus und die damit zusammenhängende Sprache der eigenen Disziplin gehören oft zum lediglich impliziten Wissen einer Person. Sie enthalten vieles, was innerhalb der eigenen Disziplin als Selbstverständlichkeit gilt. [...] Entsprechend schwierig ist es, eine gemeinsame Sprache zu finden. Konflikte entstehen, wenn die Beteiligten darauf bestehen, ihre

3 Rico Defila/Antonietta Di Giulio/Michael Scheuermann (Hrsg.): Management von Forschungsverbünden-Möglichkeiten derProfessionalisierung und Unterstützung, DFC Standpunkte, Weinheim 2008. Zu weiterer Literatur zur Problematik interdisziplinärer Kooperation vgl. S. 17. 4 Defila u.a., 2008, S. 18. 
Verwendungsweise eines Wortes sei die >richtige`, und nicht bereit sind, von ihrem Begriffsverständnis abzurücken, sondern auf einem Definitionsprimat beharren.«

Solche Konflikte betreffen nicht nur das Problem einer gemeinsamen Sprache, sondern bereits Divergenzen in der Auffassung von »Gegenstand und Fragestellung«:

»Das Wissen darüber, welche Aspekte eines (vorwissenschaftlichen) Gegenstands für eine Untersuchung relevant sind und wie sie beschrieben werden - also die Konstruktion des Forschungsgegenstands - ist Teil der disziplinenspezifischen 〉Brille`, die sich Wissenschaftlerinnen und Wissenschaftler aneignen. [...] Konflikte entstehen, wenn die Beteiligten überzeugt sind, ihre Sicht sei die einzig mögliche Sicht auf den jeweiligen (vorwissenschaftlichen) Gegenstand, sie allein würden die srichtigen und wichtigen Fragen aufwerfen und wenn sie die Fragen der anderen als weder sinnvoll noch zielführend abqualifizieren.«

Ebenso Differenzen auf der Ebene der »Methodenwahl und Wissenschaftlichkeit«:

»In der inter- oder transdisziplinären Forschung muss auf disziplinäre Methoden der Erkenntnisgewinnung zurückgegriffen werden. Die disziplinären Vorstellungen über das >richtige` Vorgehen, über Erfolg versprechende Methoden und die angemessene Art und Weise, an eine Frage heranzugehen, divergieren aber. Dasselbe gilt für die Auffassungen darüber, welche Kriterien der Wissenschaftlichkeit anzuwenden sind. [...] Konflikte entstehen, wenn sich die Beteiligten weigern, von ihren Kriterien der Wissenschaftlichkeit Abstand zu nehmen, und mit aller Macht versuchen, ihre Methoden und ihre Wissenschaftlichkeitskriterien durchzusetzen.«

Häufig spielen auch ganz grundsätzliche »Vorurteile« eine Rolle:

»Konflikte entstehen, wenn jemand besser zu wissen meint, was andere Disziplinen leisten können oder sollten, als die Angehörigen dieser Disziplinen, und wenn dies mit >Disziplinenimperialismus einhergeht, d.h. mit der Überschätzung des Beitrags der eigenen Disziplin bei gleichzeitiger Her- 
absetzung der Leistungen anderer Disziplinen (bis hin zur Negierung ihrer Wissenschaftlichkeit). $\ll^{5}$

Entsprechend hört man in interdisziplinären Forschungsverbünden hinter vorgehaltener Hand viel Unmut und Skepsis angesichts der interdisziplinären Herausforderung und es gibt offensichtlich eine verbreitete Sehnsucht, sich ins vertraute Terrain des ureigenen Materials, der beherrschten Methoden, der gewohnten Fragestellungen zurückziehen zu dürfen. Doch die Fliehkraft von sich immer weiter ins Spezielle ausdifferenzierenden Wissenschaftsdisziplinen hält im Gegenzug den ebenso starken Impuls am Leben, den Zusammenhang des Wissens wieder gewinnen zu wollen. Wie kann die eine Welt verstanden sein, wenn sie in disziplinäre Einzelwelten zerfällt, für die nicht einmal Weichenstellungen, Anschlüsse oder Übersetzungswerkzeuge in Sicht sind?

Während das frühe 19. Jahrhundert eine Vision einer Einheit des Wissens als Integral universaler Gelehrsamkeit kannte und die Phase von Mitte des 19. bis Mitte des 20. Jahrhundert als die Hochzeit der wissenschaftlichen Einheitskämpfe unter je spezifischen Deutungsansprüchen erscheint, stehen seitdem zwei Auffassungen einander gegenüber: diejenige, die Wissenschaftskulturen als je in sich motivierte, autonome Denkstile oder Wissenschaftsparadigmen sehen und diejenige, die ein Integral der Interdisziplinarität forcieren. Während die einen den Eigenwert eines jeden wissenschaftlichen Denkens großmütig in den Blick nehmen und dabei ihre Inkommensurabilität, ${ }^{6}$ also wechselseitige >Unanmessbarkeit<, konstatieren, steuern die anderen auf ein Integral zu, das stets Gefahr läuft, der pluralen wissenschaftlichen Realität ein äußerliches Programm überzustülpen.

5 Die vorangegangenen Zitate finden sich bei Defila u.a., 2008, S. 18-20.

6 Vgl. Kurt Bayertz: Das Problem der Inkommensurabilität. In: Wissenschaftstheorie und Paradigmabegriff, Stuttgart 1981, S. 77-105; Paul Hoyningen-Huene: Inkommensurabilität bei Kuhn und Theorienvergleich. In: E. Agazzi (Hrsg.): Die Vergleichbarkeit wissenschaftlicher Theorien, Freiburg 1990, S. 97-108. 


\section{Wie ist diesem Dilemma zu entkommen?}

Die Analyse von Wissenschaftstypen könnte die Antwort auf diese Frage sein. Das Stichwort heißt hier Tiefenstruktur. Wissenschaftstypen verhalten sich zu Disziplinen wie die Bewegung von Kontinentalplatten zum Oberflächenrelief. Hier wirken - um im Bild zu bleiben - die Formierungskräfte wissenschaftlicher Praxis, die die Disziplinen durchzieht. Sie bilden die Ebene, auf der sich bestimmt, was in der Forschung tatsächlich geschieht. Und zwar: was einen Forschungstyp in sich motiviert und wie er seine Frage-, Herangehens- und Darstellungsweisen ausrichtet auf das, was in ihm als Erkenntnis und Wissen gilt. Elementare Handlungsmuster wie die des Messens, Erörterns, Vergleichens, Berechnens, Mischens etc. spielen hier entscheidende Rollen. Doch auf der Ebene der Wissenschaftstypen sind sie in Motivkomplexe intergiert: man misst, um Daten zu gewinnen, die in einem bestimmten Fragekomplex und Darstellungssystem eine bestimmte instruktive Aussage z.B. in Bezug auf Klimawandel bedeuten. Oder: Man analysiert grammatische Formen, um zu untersuchen, wie ein Text sprachstrukturell von Leitgedanken seiner Epoche geprägt ist, was ein Bewusstsein für historische Spezifität schafft. Wissenschaftstypen zu beschreiben bedeutet, Praktiken in Motivationskomplexen zu erfassen. Solche Motivationskomplexe sind, wie gesehen, historisch gewachsen und prägen sich in verschiedenen Formen aus. Diese Komplexe durchziehen die Disziplinen.

Manchmal schlägt sich dies in Bezeichnungen nieder: physische Geographie versus Sozialgeographie; hypothesengeleitete Laborforschung versus Systembiologie; quantitative Soziologie versus Kultursoziologie (um einige Beispiele zu nennen). Meist aber machen sich solche Differenzen in der Tiefenstruktur nur als Spannung und Konflikt bemerkbar. Die Tiefenstruktur offenzulegen birgt die Chance in sich, ausdrücklich und aktiv mit solchen Spannungen umzugehen.

Doch wie kann auf der Ebene der Wissenschaftstypen das Problem der Interdisziplinarität gelöst werden? Wie können hier Grenzen überwunden und Forschung zusammengeführt werden? Ziel aller Interdisziplinarität ist ein Surplus, ein Synergieeffekt, der aus der Verbindung der Perspektiven entsteht. Interdisziplinarität ist nicht Selbstzweck, sondern zielt auf umfassendere Erkenntnis, auf ein breites aufgebautes Wissen. Ein Standardargument lautet: Komplexe Probleme in einer komplexen Welt erfordern komplexe Forschung. Nur: Wie kann eine solche komplexere Forschung aufgebaut 
werden? Die Forschenden wachsen in ihren Disziplinen auf, lernen in ihnen nicht nur eine Gegenstandssphäre und Methoden, sondern auch eine Sprache und - das ist vielleicht entscheidend - die Raison d'etre ihrer Forschung kennen; also: was macht sie sinnvoll, was macht sie spannend? Konfrontiert mit anderen Disziplinen, in denen eine Sprache gesprochen wird, die sich nie ganz in die eigene Forschungssprache übersetzen lässt und in Angesicht von Motiven, die quer oder schräg liegen zu denen, die die eigene Forschung ausrichten, wachsen, trotz anfänglichem Enthusiasmus, Hilflosigkeit und Frust.

Betrachtet man nun aber Wissenschaftstypen als Tiefenstrukturen von Disziplinen, dann zeigt sich eine neue Chance: die Ausrichtung der Forschung selbst, ihre Verschachtelung in Teilabschnitte und Prozessschritte wird hier zur Gemeinsamkeit; indem Forschungsmotive miteinander verknüpft und - in ihrer Gleichwertigkeit - auf verschiedene Weisen aufeinander aufgebaut werden, entstehen komplexe Forschungsformen, die in sich integriert sind. Wo tatsächlich die tiefen Differenzen liegen, da finden sich auch die Gelenkstellen, die verknüpfte Forschung mit Mehrwert erlauben.

\section{VERTIEFEND Konzepte der Interdisziplinaritätsforschung}

Um diesen Gedanken zu verdeutlichen, wird es hilfreich sein, einen Blick ins Feld von Interdisziplinaritätsforschung und Interdisziplinaritätsstudien zu werfen. Denn diese Metadisziplinen sind genau deshalb entstanden, weil sich Interdisziplinarität zwar als erstrebenswert, aber keineswegs als unproblematisch erwies. Die Fragen und die Antworten in diesem Feld betreffen daher direkt unsere Problemstellung. Die Differenzierungen, die hier vorgenommen werden, sind Versuche, das Problem in den Griff zu bekommen. Doch stimmt die These, dass das Problem der Forschungsintegration erst auf der Ebene der analysierten Wissenschaftstypen lösbar ist, dann müsste sich das auch in Bezug auf dieses Feld zeigen.

\section{Womit haben wir es zu tun?}

Interdisziplinaritätsforschung und Interdisziplinaritätsstudien haben sich seit den 1990er Jahren zu einem eigenen Forschungsfeld entwickelt und eine eigene Terminologie ausgeprägt. So unterscheidet man Multidisziplinarität, Interdisziplinarität und Transdisziplinarität. Multidisziplinär ist ein Forschungsverbund dann, wenn mehrere Disziplinen in ihm vereinigt sind, 
die jeweils ihre Perspektive auf den Forschungsgegenstand beisteuern; Interdisziplinarität setzt darüber hinaus voraus, dass die unterschiedlichen Perspektiven in eine gemeinsame Forschungsanstrengung integriert sind, was bedeutet: jede Disziplin nimmt auch andere Perspektiven auf und verändert sich in ihrer Forschungsweise; transdisziplinär heißt Forschung dann, wenn interdisziplinäre Forschungsgruppen zusätzlich außerwissenschaftliche Akteure integrieren, z.B. aus NGOs, Nutzer- und Betroffenenverbänden, Politik, Wirtschaft, Gewerkschaften etc.

Bei interdisziplinärer Verknüpfung unterscheidet man des Weiteren nach Verbünden von Disziplinen, die sich traditionell nahestehen (Narrow ID) und solchen, zwischen denen große Distanzen vermutet werden (Broad/Wide ID). ${ }^{7}$

Die interessanteste Differenzierung liegt nun aber auf der Ebene des Mediums, in dem sich Disziplinen integrieren. Ist es ein gemeinsames Problem, eine gemeinsame Theorie, ein gemeinsames Objekt oder eine gemeinsame Methode, in der sich unterschiedliche disziplinäre Forschungsperspektiven zusammenfinden? ${ }^{8}$ Der stärkste politische Impuls, der in Richtung Interdisziplinarität drängt, liegt aktuell sicherlich in der Problemorientierung: Klimawandel, Urbanisierung, Diskriminierung, Wasserknappheit, Hunger, Naturkatastrophen, Pandemien, Luftverschmutzung, Ressourcenknappheit - viele solcher Themen zeigen Problemstellungen, die an keine einzelne Disziplin delegiert werden kann. Das zentrale Argument liegt hier im Verweis auf Komplexität: Solche Probleme sind so vielgestaltig, dass sie der multiperspektivischen und zugleich integrierten Anstrengung vieler Disziplinen bedürfen.

Doch diese Differenzierung von Problem-, Objekt-, Theorie- und Methodenorientierung interdisziplinärer Forschung, die Jan C. Schmidt eindrück-

7 Julie Thompson Klein: A taxonomy of interdisciplinarity. In: Robert Frodeman/Julie Thompson Klein/Carl Mitcham/J. Brott Holbrook (Hrsg.): The Oxford Handbook of Interdisciplinarity, Oxford 2010, S. 15-30, hier S. 18.

8 Vgl. Margaret A. Boden: What is interdisciplinarity? In: R. Cunningham (Hrsg.): Interdisciplinarity and the organization of knowledge in Europe, Office for Official Publications of the European Communities, Luxembourg 1999, S. 13-24; H. Bruun,/J. I. Hukkinen/K. I. Huutoniemi/]. Thompson Klein: Promoting Interdisciplinary Research: The Case of the Academy of Finland. In: Publications of the Academy of Finland, 8 (2005); Jan C. Schmidt: Towards a philosophy of interdisciplinarity. An attempt to provide a classification and clarification. In: Poiesis and Praxis 1 (2008), S. 53-69; Jan C. Schmidt: What is a problem? On problem-oriented interdisciplinarity, Poiesis and Praxis 4 (2011), S. 249-274. 
lich ausbuchstabiert, gibt allein noch kein Werkzeug zur Integration an die Hand. Das zeigt sich, wenn wir die Orientierungen durchgehen:

Zunächst: Ein gemeinsames Problem mag in vielen Fällen Anlass sein, aber sie macht noch keine gemeinsame Forschung. Problemorientierung scheint ein viel zu breites Anliegen zu sein, um Formen wissenschaftlicher Integration daraus zu entwickeln. Übergreifende Probleme, die sich politisch artikulieren, sind zunächst nicht die Probleme, an denen sich disziplinäre Forschung ausrichtet. Problemorientierung zeigt die Notwendigkeit der Integration und gegenseitigen Übersetzung an; sie gibt aber noch kein Werkzeug an die Hand.

Kommen wir also, zweitens, zur Objektorientierung. Objektorientierung bedeutet, eine bestimmte Objektklasse zu privilegieren. Man spricht hier von einer ontologischen, also die Ordnung des Seins betreffenden Herangehensweise. Der seit der Antike verhandelte Atomismus ist eine solche Position und wir haben im vorhergehenden Abschnitt gesehen, dass es auch im 20. Jahrhundert Ansätze gab, eine Einheit der Wissenschaften durch Rückgang auf physikalische Elemente zu begründen. Der Ansatz der Unity of Science, demgemäß Geistiges, Soziales und Biologisches auf physikalische Einheiten zurückzubeziehen sei (siehe Kapitel 18) stand in dieser atomistischphysikalistischen Tradition. Für jüngere Tendenzen gibt Jan C. Schmidt ein treffendes Beispiel, wenn er einen Report der US-amerikanischen Wissenschaftspolitik zitiert, in dem Nanoobjekte als Basis einer interdisziplinären Forschungsverbundes aus Nano-, Bio-, Informations- und Kognitionswissenschaften betrachtet werden. ${ }^{9}$ Objektorientierte Interdisziplinarität zeigt sich auch hier als reduktive Strategie: Die Wahrheit der Phänomene liegt in den Eigenschaften der kleinsten Teile.

Doch wie sieht es mit Theorie- und Methodenorientierung aus? Die Idee hier ist, dass eine gemeinsame übergreifende Theorie oder eine basale Methodologie das gesuchte Integral liefern könnte. Kandidaten sind hier aufseiten der Theorie System- und Selbstorganisationstheorien, Theorien komplexer Systeme und Chaostheorien, aufseiten der Methoden mathematische Modellierungen, Bionics und ähnliches. ${ }^{10}$

9 Vgl. Schmidt, 2011, S. 266. Vgl. auch: Werner Kogge: Technologie des 21. Jahrhunderts. Perspektiven der Technikphilosophie. In: Deutsche Zeitschrift für Philosophie, 6 (2008),

S. 935-956.

10 Vgl. Schmidt, 2011, S. 254-256. 
Blickt man auf diese Kandidatenliste, so zeigt sich Theorie- und Methodenorientierung als Integral eines Forschungsprogramms, das tatsächlich den umfassendsten und aufwändigsten Ansatz darstellt, der je in Sachen interdisziplinärer Forschung veranstaltet wurde. Wenn ein Nature-Artikel von $2015^{11}$ als Institutionen mit Pioniercharakter solche aufzählt, die Informatik, Mathematik oder neurowissenschaftliche Ansätze auf andere Disziplinen anwenden (Santa Fe Institute (von 1984); Janelia Research Campus), dann ist hier zu ergänzen: Dieser Typ von Interdisziplinarität hat eine lange, starke Tradition, die die gesamte Epoche des Kalten Krieges beherrschte:

Es war der Komplex aus Kybernetik, Informationstheorie und mathematische Theorien biologischer Automaten, der seit Ende der 1940er Jahre durch eine Reihe von Publikationen Popularität erlangte: 1948 erschienen sowohl Norbert Wieners Cybernetics or control and communication in the animal and the machine und Claude Shannons The Mathematical Theory of Communication, 1949 und 1952 trat John von Neumann mit seinen Vortragsreihen The Theory and Organization of Complicated Automata und Probabilistic Logics and the Synthesis of Reliable Organisms from Unreliable Components an die Öffentlichkeit; ${ }^{12}$ des Weiteren waren die zehn interdisziplinären Macy-Konferenzen, die zwischen 1946 und 1953 stattfanden, wichtiger Katalysator für die Verschmelzung von biologischen und mathematischen Konzeptionen. Unter dem Leitbegriff $\mathrm{Cy}$ bernetics wurden Mathematik, Biologie, Computerwissenschaften, Neurologie, Maschinenlehre, aber auch Linguistik, Psychologie und Anthropologie in ein gemeinsames Feld geführt. Das generelle Thema dieser Ansätze sind Regelsysteme und die Steuerung von Verhalten; das Organische gilt dabei als Vorbild und als potenzieller Gegenstand der Entwicklung mathematischer Modelle und technischer Systeme. ${ }^{13}$ Was den Einsatz von Mitteln und die Einbeziehung von Forschungsfeldern anbelangt, ist das kybernetische Integral sicherlich das größte interdisziplinäre Forschungsprogramm, das es je gab; seine Verflechtung mit militärischen Zielvorgaben, zunächst noch im Zweitem Weltkrieg, dann im Kalten Krieg, ist viel diskutiert. Gründungen

11 Heidi Ledford: Team Science. In: Nature, 525 (2015), S. 308-311.

12 Vgl. Lily E. Kay: Das Buch des Lebens: Wer schrieb den genetischen Code?, München 2002, S. 159.

13 Vgl. Kay, 2002; Evelyn F. Keller: Das Leben neu Denken. Metaphern der Biologie im 20. Jahrhundert, München 1998; Des Weiteren: Peter Galison: Die Ontologie des Feindes. Norbert Wiener und die Vision der Kybernetik. In: Michael Hagner (Hrsg.): Ansichten der Wissenschaftsgeschichte, Frankfurt a.M. 2001, S. 433-485; Michael Hagner/Erich Hörl (Hrsg.): Die Transformation des Humanen, Frankfurt a.M. 2008. 
interdisziplinärer Institutionen wie die im Nature-Artikel zitierten stehen fast sämtlich in diesem ideellen und pragmatischen Kontext.

Mit welcher Form von Interdisziplinarität haben wir es bei Verbünden in diesem Programm zu tun? Sie ist nicht objektorientiert, denn sie ist ontologisch offen; sie ist auch nicht problemorientiert, denn die Ausrichtung auf Steuerung und Kontrolle richtet sich eher auf eine Problemlösungsstrategie als auf bestimmte Probleme. Spezifisch wird sie aber auf theoretischer und methodischer Ebene. Die Kybernetik beruht nämlich auf voraussetzungsreichen theoretischen Annahmen, die in ihrem Paradigma der Selbstorganisation impliziert sind. Das im Grunde Aristotelische Konzept (Stichwort entelecheia: Zielformation von natürlichen Wesenheiten) wurde in der Biologie des 18. Jahrhunderts (Blumenbach: Stichwort Bildungstrieb) neu aufgegriffen, in Kants Philosophie des Organischen ausbuchstabiert (Kritik der Urteilskraft; Zweiter Teil) und auf unterschiedlichen Wegen ins 20. Jahrhundert tradiert, wo es mit der mathematischen Theorie rekursiver Funktionen und neuen computertechnischen Möglichkeiten verschmolzen wurde. So entstand aus einem klassischen Theorem, das am Phänomen des Organischen gewonnen war, ein umfassendes Forschungsprogramm, das sich von der Kybernetik $\mathrm{zu}$ unterschiedlichen Ausprägungen von Systemtheorie weiterentwickelte. Paul Varelas und Hubert Maturanas Terminus der Autopoiesis (also der Selbstbildung), der grundlegend wurde für Niklas Luhmanns Theorie sozialer Systeme, spiegelt noch den Ursprung dieser theoretische Traditionslinie.

Doch lässt sich durch gemeinsame Theorie Interdisziplinarität herstellen? Kann es eine solche die Disziplinen überspannende gemeinsame Theorie überhaupt geben? Das kybernetische Paradigma ist ein Beispiel dafür, dass dies für bestimmte übergreifende Interessen wie das Bedürfnis nach Steuerung und Kontrolle möglich ist. Doch es ist auch ein Beispiel dafür, dass ein solches Paradigma von vornherein die Potentiale vieler Wissenschaften ausschließt. Denn den Gegenstand als ein rekursiv gesteuertes oder selbstorganisierendes System zu betrachten, läuft in vielen Forschungskontexten ins Leere. Das Gleiche gilt auf der Ebene der Methode für den Einsatz mathematischer Modellierung in Theorien der Selbstorganisation bis hin zu Komplexitäts- und Chaostheorien.

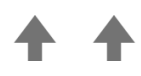


Der vertiefende Blick in die Interdisziplinaritätsforschung zeigt eine Problematik, die sich so zusammenfassen lässt:

Für die Gemeinsamkeit, die verschiedene Disziplinen in ein Forschungsvorhaben integriert, kommen vier Kandidaten infrage: eine gemeinsame Problemstellung, ein gemeinsames Objekt (Forschungsgegenstand), gemeinsame Methoden oder gemeinsame Theorien. Diese vier Kandidaten werfen jeweils eigene Probleme auf: Die gemeinsame Problemorientierung zeigt lediglich den Bedarf an interdisziplinärer Verbindung an, gibt aber noch keine Mittel an die Hand, wie eine solche zu bewerkstelligen sein kann; das Gleiche gilt für gemeinsame Gegenstände, wenn es schließlich darum geht, unterschiedliche disziplinäre Perspektiven auf ein Objekt in zusammenhängende Erkenntnis zu überführen; außerdem stellt sich die Frage, ob das vermeintlich gemeinsame Objekt in den disziplinären Perspektiven nicht völlig unterschiedlich konzeptualisiert ist - auch hier bleibt die Frage nach der Basis der Gemeinsamkeit unbeantwortet. Gemeinsame Methoden und gemeinsame Theorien auf der anderen Seite werfen die Frage auf, ob es sich überhaupt um unterschiedliche Disziplinen handeln kann, wenn sie in einem einzigen methodisch-theoretischen Horizont arbeiten. Denkbar ist so etwas in einer Art Meta-Methodik und Meta-Theorie. Und genau dafür gibt es im 20. Jahrhundert ein Beispiel, das das größte und vielleicht auch einzige umfassend realisierte Forschungsprogramm methodischer und theoretischer Interdisziplinarität darstellt: Von den 1950er bis in die 1990er Jahre bildeten die Theorien von Steuerung, Selbstorganisation und Ordnungsbildung ein theoretisches Dach, das methodisch mit mathematischer und informationstechnischer Modellierung arbeitete. Kybernetik, Systemtheorie, Komplexitätstheorie, Chaostheorie, mathematische Modellierung und Fuzzy Logic sind Titel für Teile und Stufen dieses Forschungsprogramms. ${ }^{14}$

Jedoch: Die Integration in eine Methodik, die Phänomenbereiche mathematisch-formalistisch zu fassen sucht, ist, wie wir sehen konnten, nur ein bestimmter Typus von Wissenschaft. Für viele Forschungsgegenstände ist er ungeeignet und vielen Disziplinen fremd. Wir haben es also weniger mit einer Anleitung zu interdisziplinärer Kooperation denn mit einer Übersetzung in

14 Damit ist nicht gesagt, dass sich nicht jüngere Ansätze von Systemtheorien diesem Modell auch entziehen; Es wäre aber zu prüfen, ob sie sich damit gegenüber dem ursprünglichen Forschungsprogramm nicht isolieren; ein zu prüfendes Beispiel wäre etwa die soziologische Systemtheorie nach Niklas Luhmann. 
ein spezifisches Forschungsprogramm zu tun. Ebenso ist das theoretische Erkenntnisinteresse der Steuerung komplexer, dynamischer Ordnungen - so allgemein es gehalten ist - doch ein spezifisches. Für viele wissenschaftliche Fragestellungen spielt dieser Theorieansatz überhaupt keine Rolle.

Mit diesen Überlegungen deutet sich an, dass die Frage der Integration von Erkenntnissen als Frage der Interdisziplinarität noch nicht tief genug gestellt ist. Disziplinen sind als Produkte vielfältiger historischer Entwicklungen in sich selbst methodisch, theoretisch und gegenständlich divers und untereinander undeutlich abgegrenzt. Die wirksamen Unterschiede liegen auf der Ebene der Wissenschaftstypen, die die Disziplinen durchziehen.

Auf dieser Ebene der Wissenschaftstypen zeigt sich nun aber, dass die Suche nach einer gemeinsamen Basis für integriertes Arbeiten eine letztlich unmöglich zu realisierende und auch unnötige Voraussetzung macht: nämlich, dass Integration genau da und so weit gelingt, wie Gleichheit vorausgesetzt oder Angleichung durchgesetzt werden kann.

Mit dem Theorem der Familienähnlichkeiten (der Wissenschaftstypen) kommt hier eine neue Denkfigur zum Vorschein: die Figur der ergänzenden und fortführenden Verknüpfung selbständiger unterschiedlicher Einheiten anstelle der Figur der einheitlichen Basis und Angleichung.

\section{Noch einmal zum Konzept der Wissenschaftstypen: Idealtypen mit Anschlussstellen. Ein modulares Konzept interdisziplinärer Zusammenarbeit}

Wissenschaftstypen als historisch gewachsene Motivkomplexe zu analysieren, bedeutet auch, sie jeweils als eigenständig und eigenwertig zu betrachten. In ihnen selbst liegt das Potential, gemäßihrer Motive validiertes Wissen zu produzieren, also solches, das sich an Wertungskriterien der jeweiligen Wissenschaft bemisst. Schaut man aber auf Wissenschaftstypen vor dem Hintergrund der Interdisziplinaritätsthematik, dann stellt sich die Frage, ob Wissenschaftstypen Solitäre sind, die sich der Zusammenarbeit untereinander entziehen. Einen Gedanken zur Auflösung der Spannung zwischen Autonomie und Zusammenhang haben wir im Kapitel 20 angesprochen: das Wittgensteinsche Theorem der Familienähnlichkeiten, mit der Metapher des Taus, in dem sich viele einzelne Fasern überlappen und zu einem starken Tau verbinden, ohne dass eine einzelne Faser als die sallgemeine das gesamte 
Tau durchzieht. Dieses Konzept, das einen neuartigen Begriff vom Begriff hervorbrachte, hat befreiende Wirkung: Es entbindet von der Aufgabe, die allgemeinen definitorischen Eigenschaften von Wissenschaft aufzusuchen, ein Unterfangen, das in sich stets die Tendenz birgt, partielle Sichtweisen mit hegemonialen Ansprüchen hervorzubringen.

Eine anti-hierarchische Sicht auf die Pluralität der Wissenschaften findet im Theorem der Familienähnlichkeit eine gute Grundlage. Was allerdings in diesem Theorem noch nicht ausgedrückt ist, ist die Antwort auf die Frage, wie die Verknüpfung der Einzeltypen vonstattengehen kann. An dieser Stelle steht im Wittgensteinschen Kontext das Konzept der Analogie. Analogie (ursprünglich ein Begriff der Aristotelischen Metapherntheorie) bezeichnet im Kern, dass sich durch die Entdeckung gemeinsamer Eigenschaften sehr unterschiedlich erscheinende Gegenstände miteinander ins Verhältnis stellen lassen und sich damit zeigt, wie sie zueinanderstehen.

Die unterschiedlichen Wissenschaftstypen sind ein Anwendungsfall analogischer Verknüpfung. In ihren Eigenschaften treten unterschiedliche Gemeinsamkeiten und partielle Überlappungen auf. Diese jeweils anderen Gemeinsamkeiten bilden, entdeckt man sie und legt sie frei, die Andockstellen für wissenschaftliche Kooperation.

Um diesen Gedanken zu verdeutlichen, ist es hilfreich, ein weiteres Theorem aufzurufen: Max Webers Konzept der Idealtypen. Idealtypen lassen sich als paradigmatische, also mustergültige Fälle begreifen, die tatsächlich vorkommen, aber selten in Reinform, und die vor allem als ein Vergleichsobjekt dienen, um eine Vielfalt von Phänomenen beschreiben zu können - kontrastiv. Wissenschaftstypen sind solche Idealtypen. Es gibt Fälle, in denen wissenschaftliche Forschungsprojekte vollständig innerhalb eines wissenschaftlichen Typus angelegt sind. Für gewöhnlich ist Forschungspraxis aber ein Tätigkeitskomplex, in dem sich unterschiedliche typische Modalitäten kombiniert finden. Dies geschieht unter einem Leitmotiv, sodass sich verschiedenartige wissenschaftstypische Fragen und Motive häufig im Gesamtrahmen eingeschachtelt finden.

Und es scheint fast so, als ob solche Einschachtelungen von Wissenschaftstypen sich restlos als Stationen in wissenschaftlichen Forschungsprozessen verstehen lassen (vgl. Abb. 14). Spielen wir das einmal durch: (1) Man beobachtet, vermisst, beschreibt einen Gegenstand, sodann (2) vergleicht man ihn, ordnet ihm einen Typus, einem Genre, einer Epoche zu, klassifiziert ihn, bestimmt (3) die Häufigkeit seines Auftretens in Bezug auf be- 
stimmte Korrelationen, somit die empirische Vorkommensverteilung; setzt dann mit einer Interpretation an, in der das Phänomen im Zusammenhang von Teil und Ganzem rekonstruiert wird, wobei der Schwerpunkt (4) auf der Synthese von Anzeichen, Spuren, Relikten oder (5) auf der Analyse möglicher Bedingungen liegen kann, (6) arbeitet man daran, Begriffe, Interpretationen, Modelle konzeptuell anzupassen, (7) werden kritisch die Bedingungen reflektiert, unter denen Gegenstandsbestimmungen, Typisierung, Statistik und Interpretation zustande gekommen sind, was mit Aussagen über Reichweite der Erkenntnisse und über notwendige weitere Forschungen und veränderte Programme verbunden ist; (8) reduziert man den interpretierten Gesamtzusammenhang auf ein universales Regelwerk, das sich idealiter in einer formalen Sprache ausdrücken lässt, oder versucht (9) das verhandelte Phänomen unter Laborbedingen zu reproduzieren (um schließlich zu einer praktisch-technischen Anwendung zu kommen).

Man könnte so zu einem Bild kommen, demgemäß die Wissenschaftstypen nicht wirklich separate und autonome Forschungseinheiten darstellen, sondern unterschiedliche Schritte oder Stationen in einem einheitlichen Forschungszusammenhang. Ein Verlaufsdiagramm von Forschung könnte demgemäß so aussehen:

Betrachten wir aber die Rhetorik, in der wir dieses Ablaufschema entworfen haben und die Bildlogik, in der wir es dargestellt haben, dann zeigt sich die Suggestion, mit der ein solches Schema stets verbunden ist: Das Handeln scheint hier von anfänglichen, elementaren Schritten zu hochkomplexen und subtil-reflektierten Tätigkeiten fortzuschreiten. Es scheint, als ob wir von der Kärrnerarbeit im Heizkeller der Forschung immer höher in die sauberen Tätigkeiten der Weißkittel-Etagen aufsteigen. Wenn diese shöheren< Tätigkeiten dann auch noch in hoch-artifiziellen und technisch voraussetzungsreichen Sprachen wie etwa der shöheren Mathematik oder der poststrukturalistischen Philosophie ausgedrückt werden, dann scheint es, als ob es sich in diesem Prozess um einen Gang von primitiven Vorarbeiten zu elaborierter Erkenntnis handelt.

Doch das ist, wie gesagt: alles Suggestion; geschuldet bestimmten Vorurteilen darüber, was wissenschaftliche Erkenntnis im Kern letztlich ausmacht; Quelle all der Status- und Deutungskämpfe, die sich durch die Geschichten der Wissenschaften ziehen.

Dass die Verschachtelung der Wissenschaftsmotive und Fragestellungen nicht als ein lineares Aufstiegsschema verstanden werden kann, dass es sich 


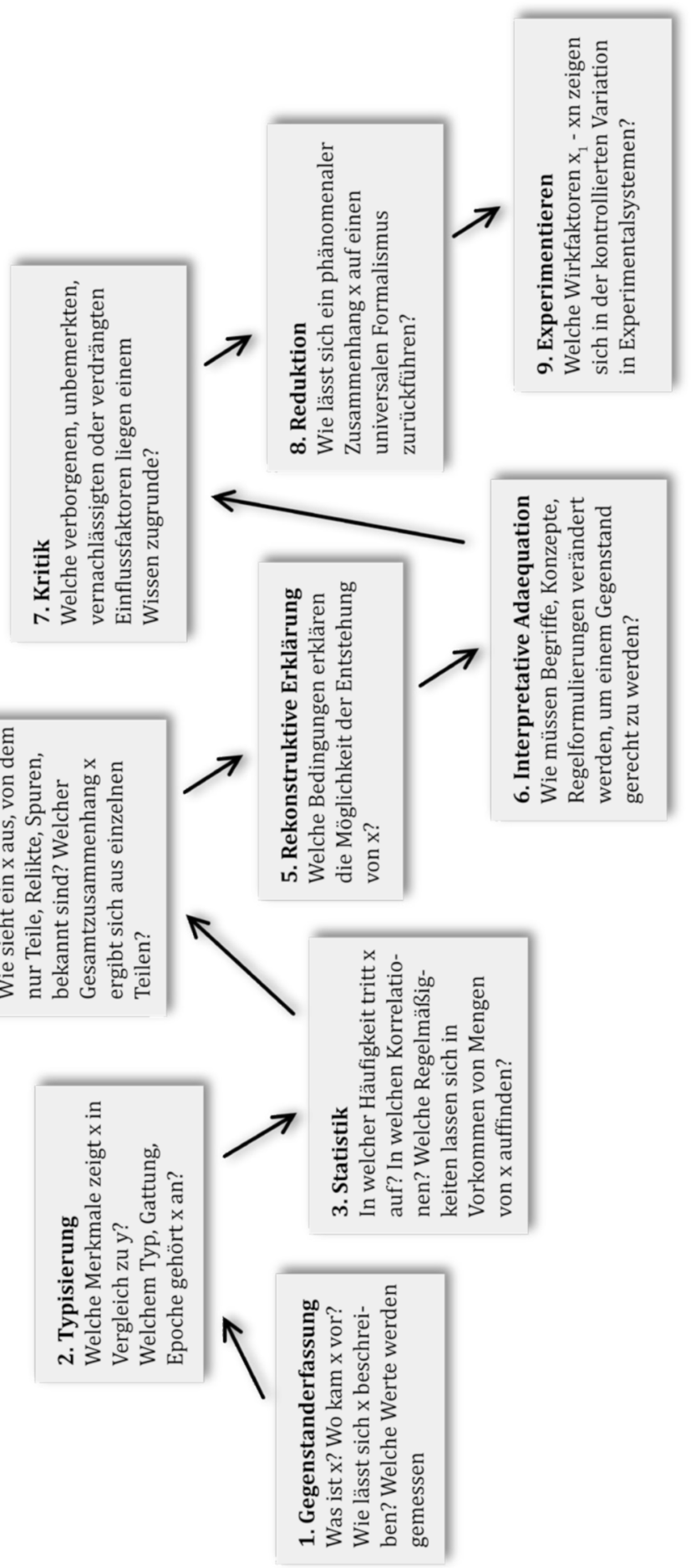


vielmehr um ein modulares System handelt, in dem sich Forschungsprozesse auf verschiedene Weisen kombiniert finden, offenbart sich nämlich in der Tatsache, dass man die Richtungen umkehren kann (vgl. Abb. 15). So gibt es Forschungsprojekte, in denen Bedingungen rekonstruiert werden (5), um zu einer Typisierung (2) zu gelangen; Laborversuche (6) werden angestellt, um Phänomene z.B. chronologisch oder geographisch (1) verorten zu können; unbefragte Voraussetzungen von Forschungsprogrammen werden befragt (7), was zu einer Neubeschreibung des Gegenstandes (1) führt.

Um der Suggestion zu entkommen, ist es wiederum hilfreich, die Darstellungs- und Bildlogik in eine konsequent-flächige zu transformieren, in der auf einer Ebene unterschiedliche Verknüpfungsformen gleichwertig erscheinen.

Schon diese Andeutungen weisen darauf hin, dass jeder Wissenschaftstyp das Erkenntnisziel eines konkreten Forschungsprogramms bestimmen kann. Das Motiv eines jeden Typus kann die Zielstellung der Arbeit ausmachen, sodass wir zu einem Bild der Kombinierbarkeit kommen, eine egalitäre Modularität sozusagen.

\section{Egalitäre Modularität: Was ist damit gemeint?}

Es bedeutet, dass typische wissenschaftliche Praxis-Motiv-Komplexe sich als Module auffassen lassen, das heißt als selbständige Funktionseinheiten, die in einem komplexen Ganzen integriert sind. Das komplexe Ganze ist hier ein konkretes Forschungsprojekt. Solche Forschungsprojekte sind typischerweise modular aufgebaut. Allerdings wird dieser modulare Aufbau selten als solcher wahrgenommen. In den Projektbeschreibungen verschwindet er unter Rubriken wie Vorarbeiten und Forschungsprogramm. Was wäre, wenn dies nicht geschähe? Wie sähe dies aus?

Wären Forschungsprojekte so zu entwerfen, dass ihr modularer Aufbau expliziert werden würde, dann hätte dies eine ganze Reihe von Effekten zur Folge:

1. Der Eigenwert der Forschungsschritte wird sichtbar, das heißt jedes solches Element wird als eigener Typus von Wissenschaft Beachtung finden, was die Sorgfalt der Planung, Ergebnissicherung usw. befördern kann;

2. es wird sichtbar, dass bestimmte modulare Einheiten, wie z.B. eine kritische Reflexion oder eine typisierende Einordnung in einem Projekt fehlt, obwohl es für die Gesamtfragestellung erforderlich sein könnte; 


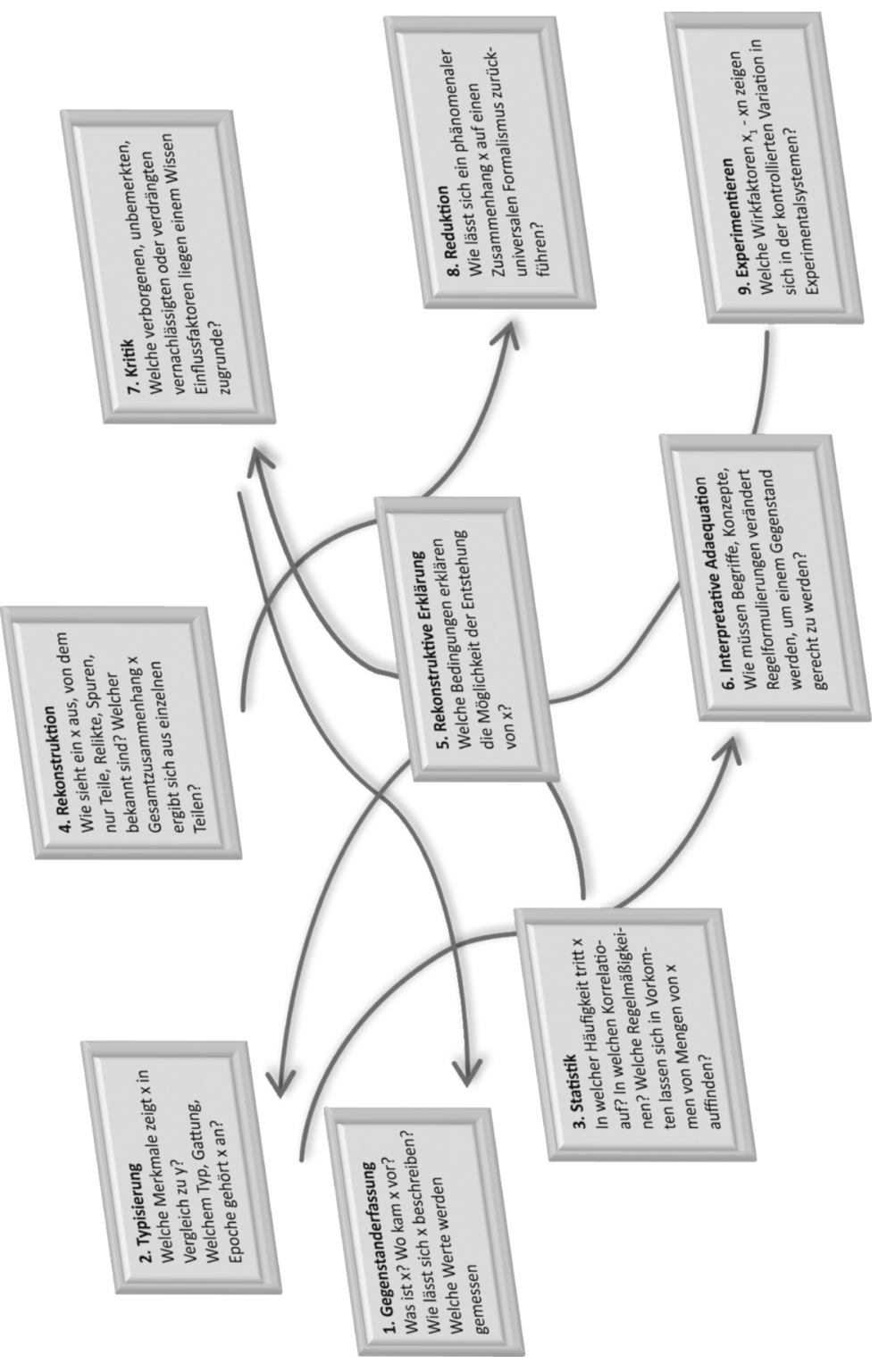




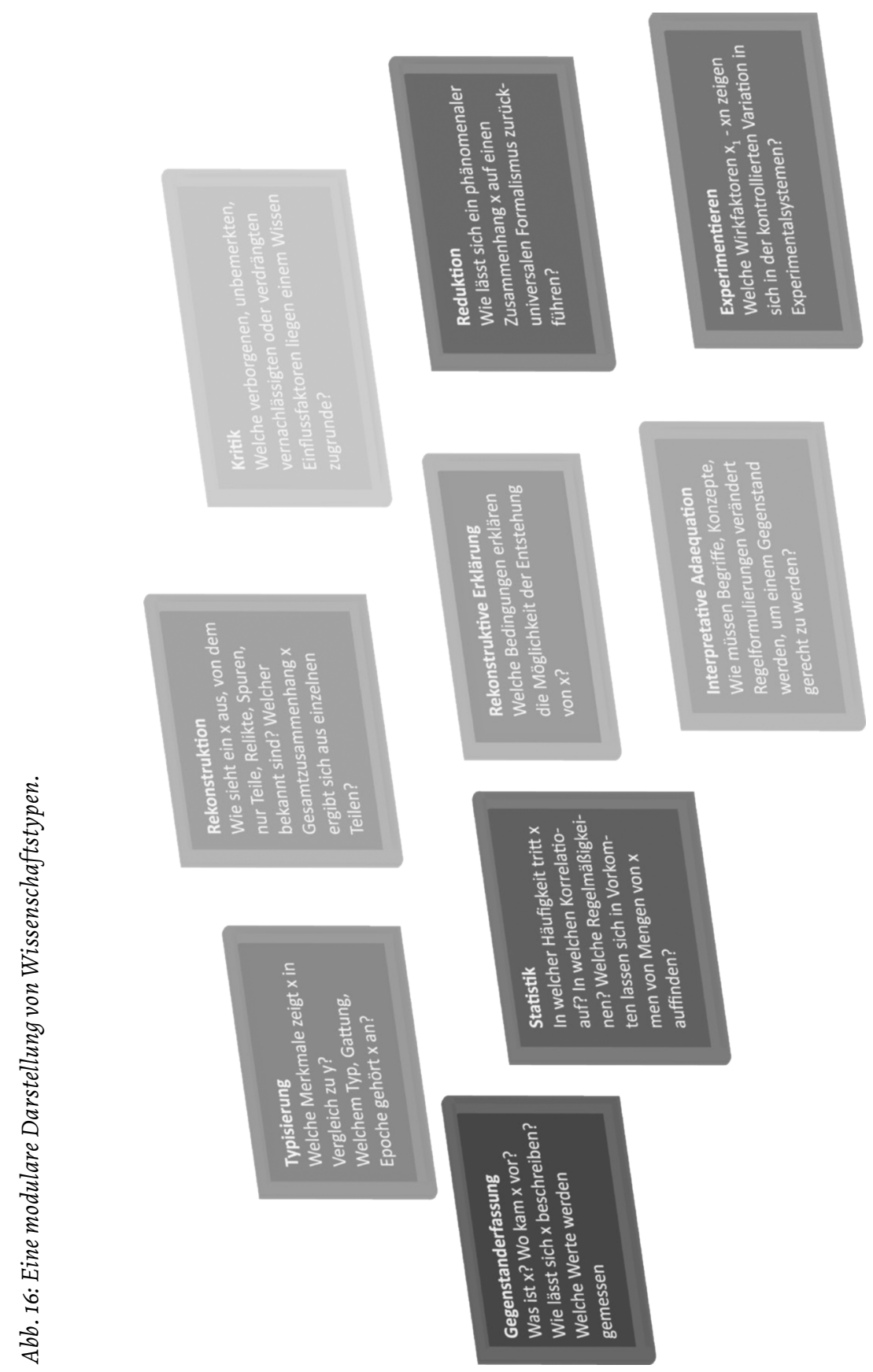


3. Begründungen, welche Module wie miteinander verknüpft werden, werden explizit, damit reflektier- und verhandelbar.

Insgesamt würde eine solche modulare Sichtweise dazu führen, Forschungsprojekte weitaus bewusster $\mathrm{zu}$ entwerfen. Man könnte sagen: die Gestaltungstiefe von Forschungsprojekten reicht dann viel tiefer; und zwar bis zur Ebene der Strukturen, in denen ihre Praxen wurzeln und motiviert sind.

Der Aufbau von Forschungsprojekten aus eigenständigen Modulen impliziert eine ganz neue Tätigkeit im Forschungsdesign: Planung bedeutet hier auch eine elementare Kombinatorik. Es geht um die Auswahl der Modulelemente - welche sind wichtig? - und um ihre Anordnung. Dass Forschungsprozesse motiviert sind, und dass sie Zielstellungen enthalten, die jeweils durch die übergreifende Fragestellung eines Wissenschaftstypus geprägt sind, dieser Aspekt bleibt erhalten. Was sich ändert, ist zweierlei: zum einen wird klar, dass jede übergreifende wissenschaftstypische Leitfragestellung eine optionale Wahl ist. Das heißt, sie ist nicht dadurch bedingt, dass ein wissenschaftliches Ergebnis diese oder jene typische Form hat. Die Starrheit, die die wissenschaftlichen Deutungskämpfe in die Forschungen tragen, wird somit überwunden. Zum anderen steht stets infrage, und erfordert eine Begründung, ob bestimmte Module in ein Forschungsprojekt eingebaut oder nicht eingebaut werden. Nicht jedes Forschungsprojekt muss aufgeblasen werden, um die Gesamtheit der modularen Elemente zu integrieren. Aber in der Forschungsplanung die Liste der Module durchzugehen und darzulegen, welche Rolle sie jeweils im konkreten Projekt spielt oder nicht spielt, das kann zu einer entscheidend vertieften und weit umfassender reflektierten Forschungsplanung führen. Das schließt Fragen ein wie diejenige, welche Forschungsansätze in Ergänzung mit- und zueinander stehen sollten und wie unterschiedliche Forschungsansätze aufeinander abgestimmt werden.

Schauen wir uns exemplarisch drei sehr einfache Beispiele für die modulare Anlage von Forschungsprojekten an. (Abb. 17)

Diese einfachen zweischritten Anlagen von Forschungsprojekten sind der Übersichtlichkeit der Darstellung geschuldet. Reale Forschungsprojekte werden im Durchschnitt eine komplexere Modularität aufweisen, also aus der Kombinatorik von mehreren Wissenschaftstypus-Bausteinen bestehen.

Eine modulare Anlage von Forschungsplanung bedeutet auch ein neu ausgerichtetes Programm von Interdisziplinarität. Denn wenn in der Forschungsplanung bestimmte Module als sinnvoll erachtet und eingebaut 


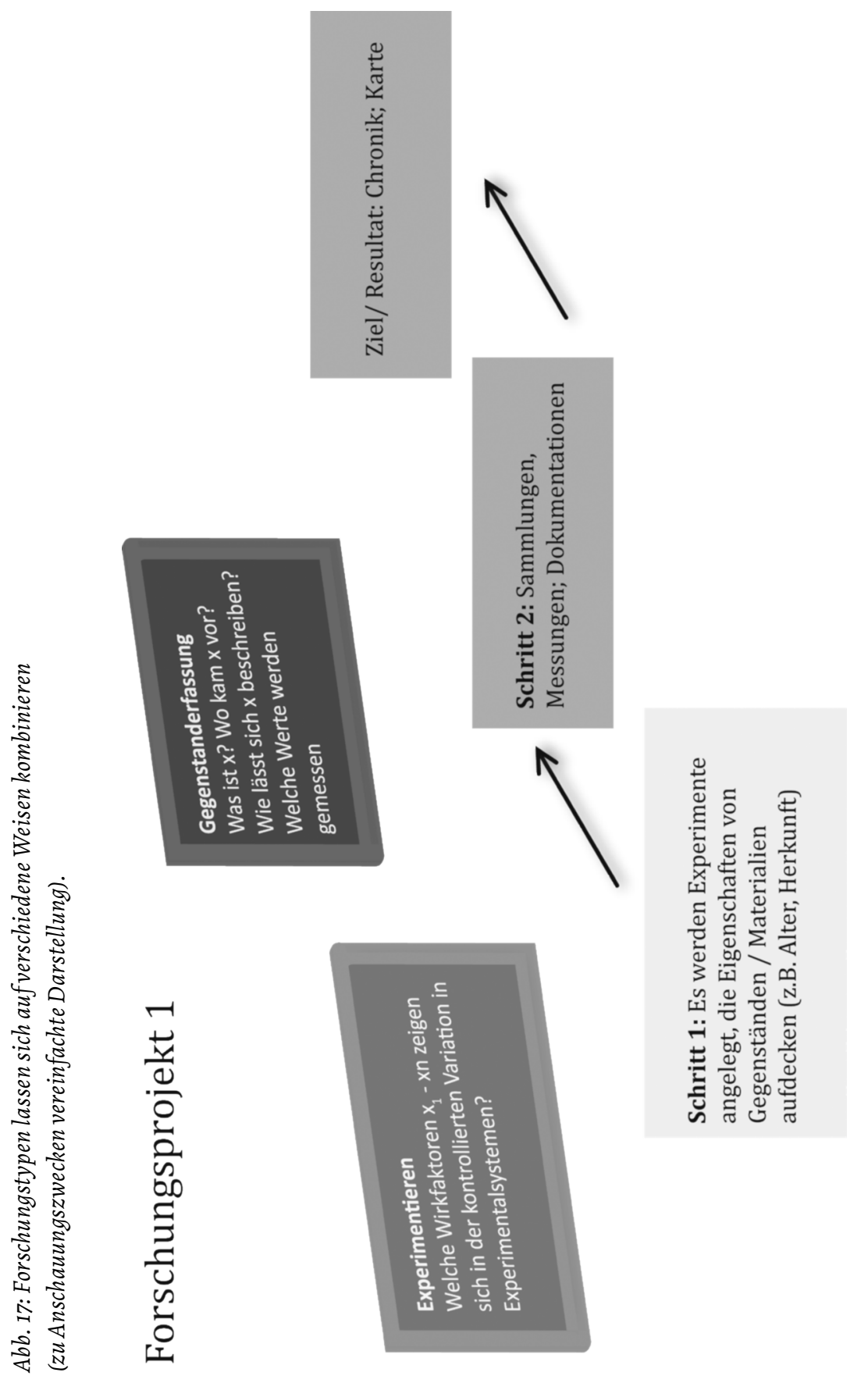




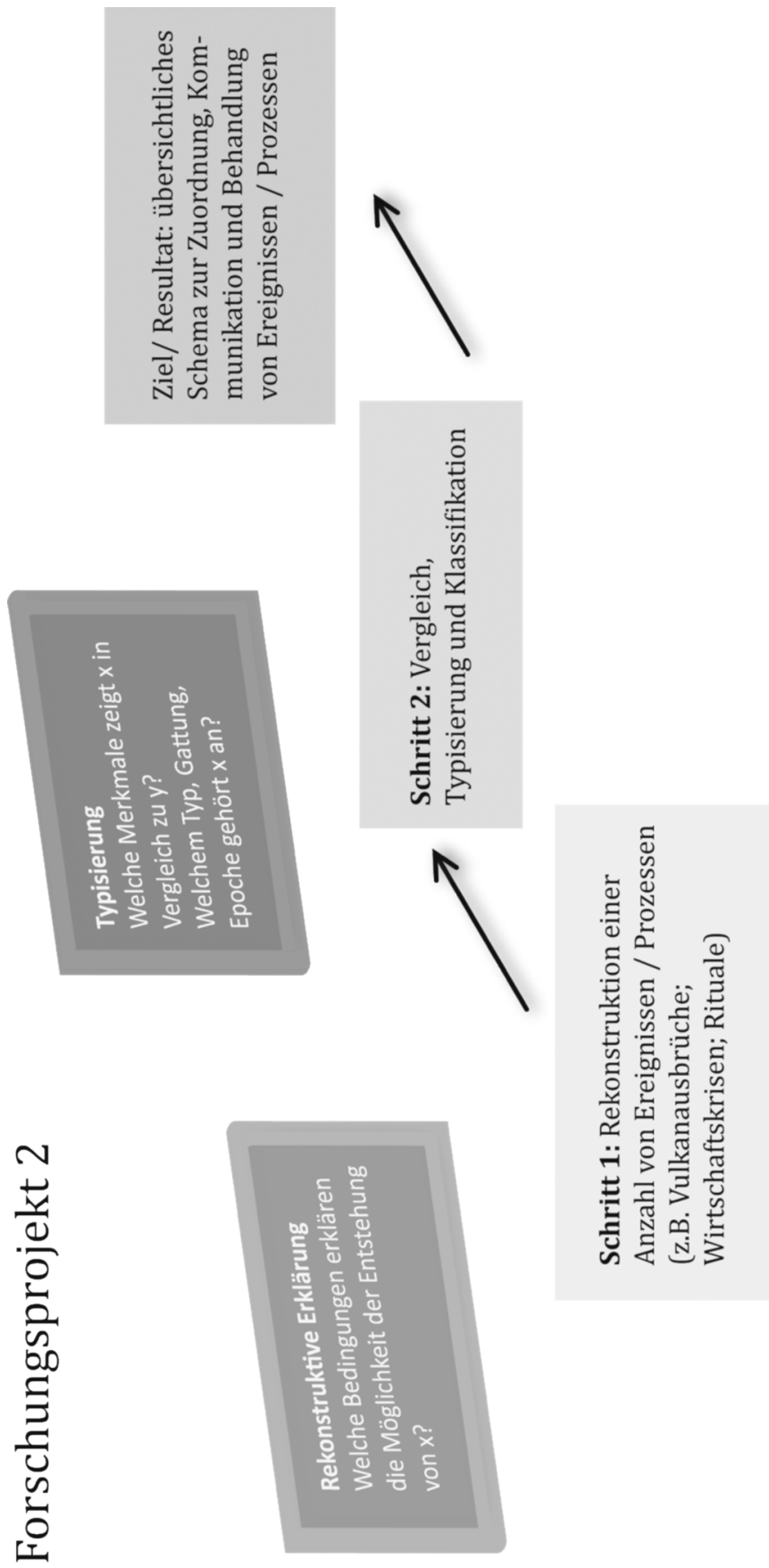




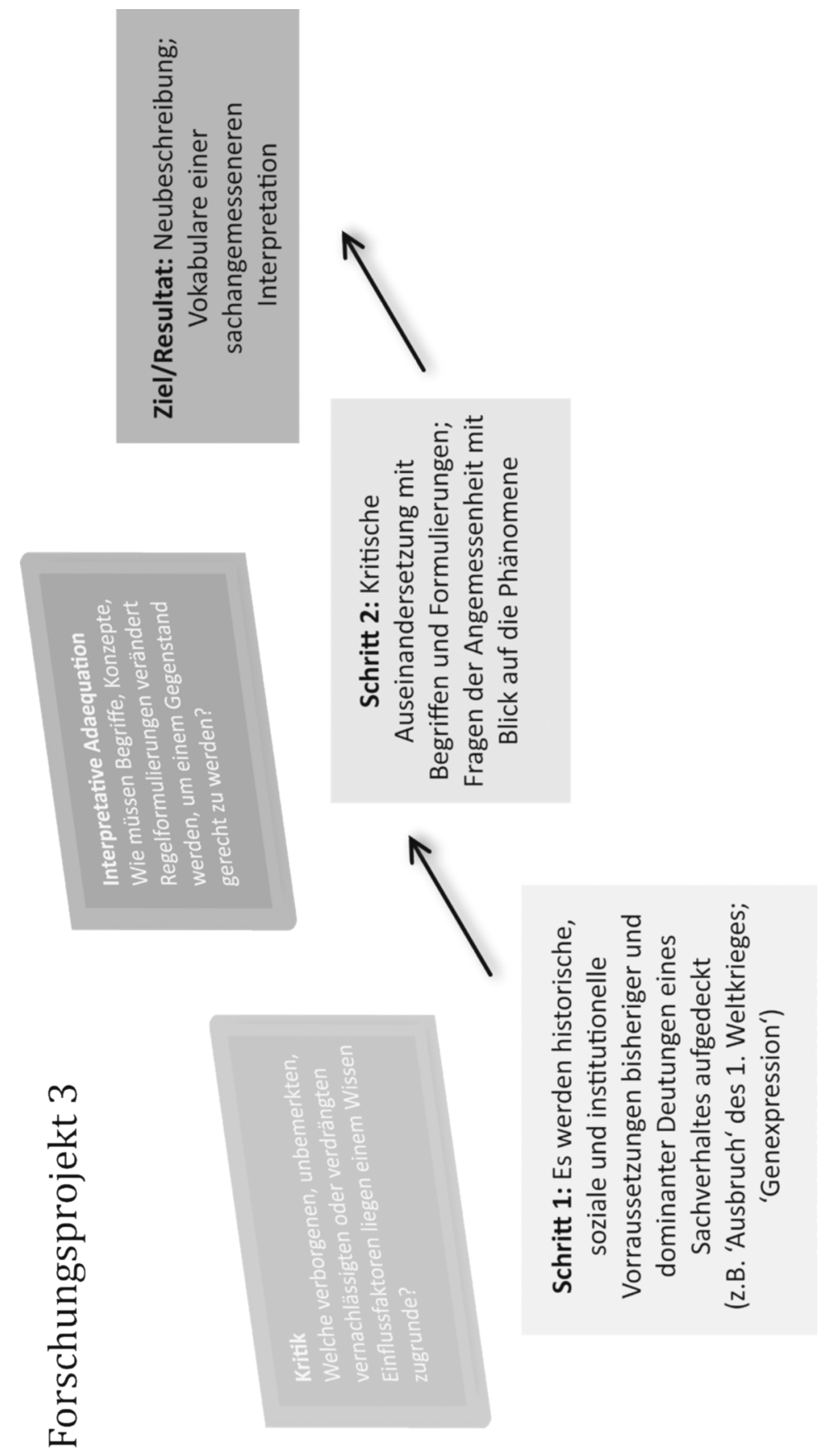


werden, dann lässt sich zielgenau danach fragen, in welchen Disziplinen die geforderten Kompetenzen zu finden sind. Die interdisziplinäre Integration wird hier, anders als bisher, nicht durch eine äußerliche Problemstellung oder durch Strategien der mehr oder weniger verdeckten Reduktion auf spezifische Objektklassen, Theorien oder Methoden vollzogen. Modulare Interdisziplinarität ist radikal nicht reduktiv. Zwar bleibt die Integration in eine übergreifende Leitfragestellung erhalten, doch behalten zum einen Module als komplette Funktionseinheiten ihren Eigenwert und zum anderen lässt sich die Leitfragestellung austauschen. Das heißt, dass die gleiche Forschungsprogrammatik in unterschiedlicher Ausrichtung mehrfach durchlaufen werden kann. Das könnte außerordentlich interessante Ergebnisse liefern und böte zudem die Chance, die Effektivität von Forschungsprogrammen zu erhöhen.

Was in der Logik dieser Überlegungen nicht möglich erscheint, ist eine Forschungsprogrammatik ohne Zielstellung. Ein solch anarchisches Forschen mag als Idee attraktiv erscheinen, ${ }^{15}$ aber es widerspricht der Grundannahme, dass Forschung als Praxis eingebettet in Motivationen arbeitet. Es liegt tief in den historisch gewachsenen Wissenschaftstypen motiviert, was, wie und woraufhin etwas untersucht wird. Motivation bedeutet Ausrichtung. Und wie es zwar immer möglich ist, nacheinander in unterschiedliche

\section{Abb. 18: Aspektwechsel-der Hasen-Enten-Kopf.}

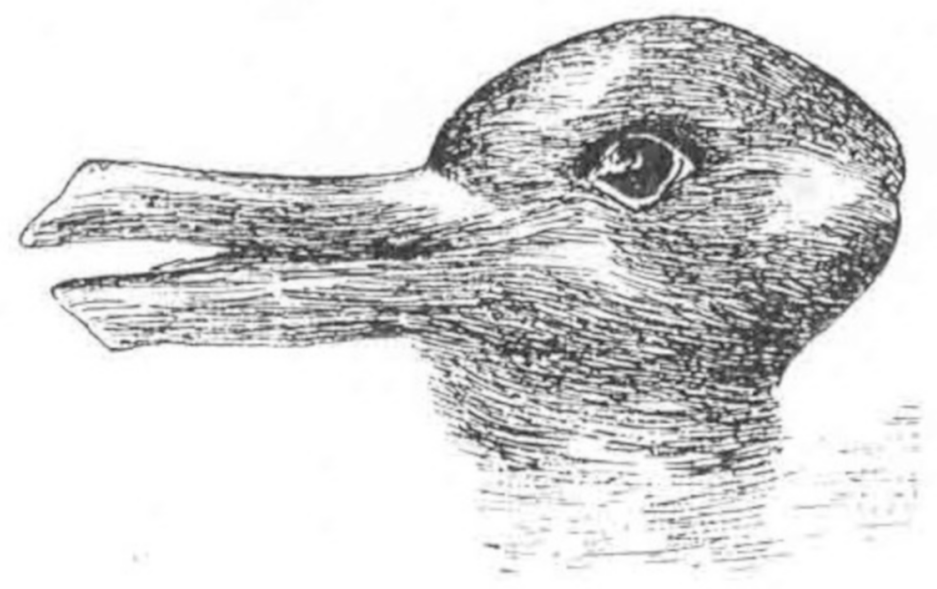

15 Vgl. Paul Feyerabend: Wider den Methodenzwang, Frankfurt a.M. 1983 (1975). 
Richtungen zu gehen und unterschiedliche Ziele anzusteuern, aber nicht zugleich, so bedeutet der modulare Aufbau die Möglichkeit einer alternierenden Zielstellung, nicht aber die Auflösung in ein ranything goes zu jeder Zeit. Wir haben es hier mit der phänomenalen Struktur des Aspektwechsels zu tun. Etwas lässt sich auf die eine und auf andere Arten ansehen; aber nicht zur gleichen Zeit. Die Blickweise kann einmal in der einen Weise ausgerichtet werden, dann wieder in der anderen. Jeweils wird etwas sichtbar, was in der anderen Blickweise verschwindet. Beide Blickweisen betrachten das Ganze, keine ist reduktiv, keine setzt bestimmte Elemente als Letzteinheiten oder bestimmte Strukturen als >die wahre Gestalt‘.

Die Analogie zum visuellen Aspektwechsel trägt noch einen Schritt weiter: Aspektwechsel kann nicht technisch erlernt werden - es gibt dazu keine Methode -; er kann aber geübt werden. ${ }^{16}$ So schult auch eine modulare Form von Interdisziplinarität auf der Tiefenstrukturebene von Wissenschaftstypen eine bestimmte Fähigkeit: Die Fähigkeit, die Blickrichtung zu ändern, bedeutet hier die Kompetenz, Forschung auf verschiedene Weisen anlegen zu können. Auf diese Weise kann erreicht werden, dass Forschungsplanung kreativer und freier und zugleich strukturell tiefer angelegt werden kann.

Spannungen zwischen den Wissenschaftstypen verschwinden auch in diesem modularen Bild wissenschaftlicher Kooperation nicht ganz. Die Auseinandersetzung um Fragen der Art, ob hinter allen Phänomenen eine formal erfassbare Weltordnung steht oder ob jedes individuelle Geschehen eine eigene Ordnung darstellt, die nur rekonstruiert oder beschrieben werden kann; der Streit, ob wir durch alle Bedingtheit unserer Erkenntnisse auf die Realität hindurchsehen können oder ob wir überhaupt nur so viel wahrnehmen, wie wir in seinen Bedingtheiten reflektieren; solche Konflikte werden nicht einfach verschwinden. Doch auch solche Konflikte erscheinen in einem anderen Licht, wenn unterschiedliche Wissenschaftstypen als gleichberechtigt anerkannt werden, und wenn Zusammenarbeit heißt: die unterschiedlichen Typen stark zu machen - stark als eigenständige Module in integrierter Forschung.

16 Vgl. dazu Werner Kogge: Das tätige Auge des Denkens: Aspektwechsel bei Wittgenstein und Fleck. In: Birgit Griesecke (Hrsg.): Werkstätten des Möglichen 1936. L. Fleck, E. Husserl., R. Musil, L. Wittgenstein, Würzburg 2008, S. 59-75. 